\title{
Association of ventricular dyssynchrony and strain with cardiac function in patients with repaired tetralogy of Fallot
}

\author{
Linyuan Jing ${ }^{1,3^{*}}$, Jonathan D Suever ${ }^{1,3}$, Richard Charnigo ${ }^{2}$, Sudad Al hadad ${ }^{3}$, Evan Stearns ${ }^{3}$, Dimitri Mojsejenko ${ }^{3}$, \\ Christopher M Haggerty ${ }^{1,3}$, Kelsey Hickey ${ }^{4}$, Anne Marie Valente ${ }^{4}$, Tal Geva ${ }^{4}$, Andrew J Powell ${ }^{4}$, \\ Brandon K Fornwalt ${ }^{1,3}$
}

From 19th Annual SCMR Scientific Sessions

Los Angeles, CA, USA. 27-30 January 2016

\section{Background}

Patients with repaired tetralogy of Fallot (rTOF) have impairments in cardiac mechanics (strain and dyssynchrony). However, the relationship between cardiac strain, dyssynchrony, and cardiac function (measured by ejection fraction) in patients with rTOF has not been explored comprehensively in a large population. We hypothesized that measures of cardiac mechanics are associated with cardiac function in patients with rTOF.

\section{Methods}

A database search identified patients with rTOF at a single institution who underwent cardiac MRI from May 2005 to March 2012. Left and right ventricular (LV and RV) ejection fraction (EF) were quantified from the MRI and used as measures of cardiac function. Seven parameters of cardiac mechanics were computed using a custom feature-tracking algorithm: LV, RV and interventricular dyssynchrony, as well as LV and RV peak circumferential and longitudinal strains. Dyssynchrony measures and peak circumferential strains were quantified from a stack of short-axis images covering both ventricles, and peak longitudinal strains were quantified from a long-axis four-chamber view. All measures of dyssynchrony were reported as positive numbers, with larger values representing more dyssynchrony. Peak circumferential and longitudinal strains were reported as absolute percentage. A linear regression model was used

${ }^{1}$ Institute for Advanced Application, Geisinger Health System, Danville, PA, USA

Full list of author information is available at the end of the article to determine the correlation between measures of cardiac mechanics and ventricular function.

\section{Results}

153 patients with rTOF ( $23 \pm 14$ years, $50 \%$ male) were included. LV circumferential strain was strongly correlated with LV EF $(\mathrm{r}=0.73, \mathrm{p}<0.001)$ and moderately with RV EF ( $r=0.43, p<0.001)$ (Figure). Similarly, RV circumferential strain correlated with RV EF $(r=0.70, p$ $<0.001)$ and LV EF $(\mathrm{r}=0.46, \mathrm{p}<0.001)$ (Figure). Both LV and RV longitudinal strains were moderately correlated with LV and RV EF (Table 1). Intra-ventricular (both LV and RV) dyssynchrony was weakly correlated with LV and RV EF (all $|r| \leq 0.26)$ (Table). Inter-ventricular dyssynchrony was modestly correlated with RV EF $(\mathrm{r}=-0.41, \mathrm{p}<0.001)$, but not with LV EF $(\mathrm{r}=-0.04$, $\mathrm{p}=0.58)$.

\section{Conclusions}

In patients with rTOF, measures of ventricular dyssynchrony and strain are associated with ventricular function measured by ejection fraction. The correlations between LV mechanics and RV function (and vice versa) may indicate unfavorable ventricular-ventricular interaction. The weak association between ventricular dyssynchrony and cardiac function suggests that dyssynchrony and dysfunction may occur independently in patients with rTOF. 
Table 1 Summary of associations between cardiac dyssynchrony/strain and ventricular function

\begin{tabular}{ccccc}
\hline & & RVEF (\%) & & LV EF (\%) \\
\hline & $\mathbf{r}$ & $\mathbf{p}$ & $\mathbf{r}$ & $\mathbf{p}$ \\
\hline LV dyssynchrony (ms) & -0.17 & 0.03 & -0.26 & $<0.001$ \\
RV dyssynchrony (ms) & -0.20 & 0.01 & -0.21 & 0.008 \\
Inter-V dyssynchrony (ms) & -0.41 & $<0.001$ & -0.04 & 0.58 \\
LV circ. strain (\%) & 0.43 & $<0.001$ & 0.73 & $<0.001$ \\
RV circ. strain (\%) & 0.70 & $<0.001$ & 0.46 & $<0.001$ \\
LV long. strain (\%) & 0.46 & $<0.001$ & 0.47 & $<0.001$ \\
RV long. strain (\%) & 0.46 & $<0.001$ & 0.35 & $<0.001$ \\
\hline
\end{tabular}
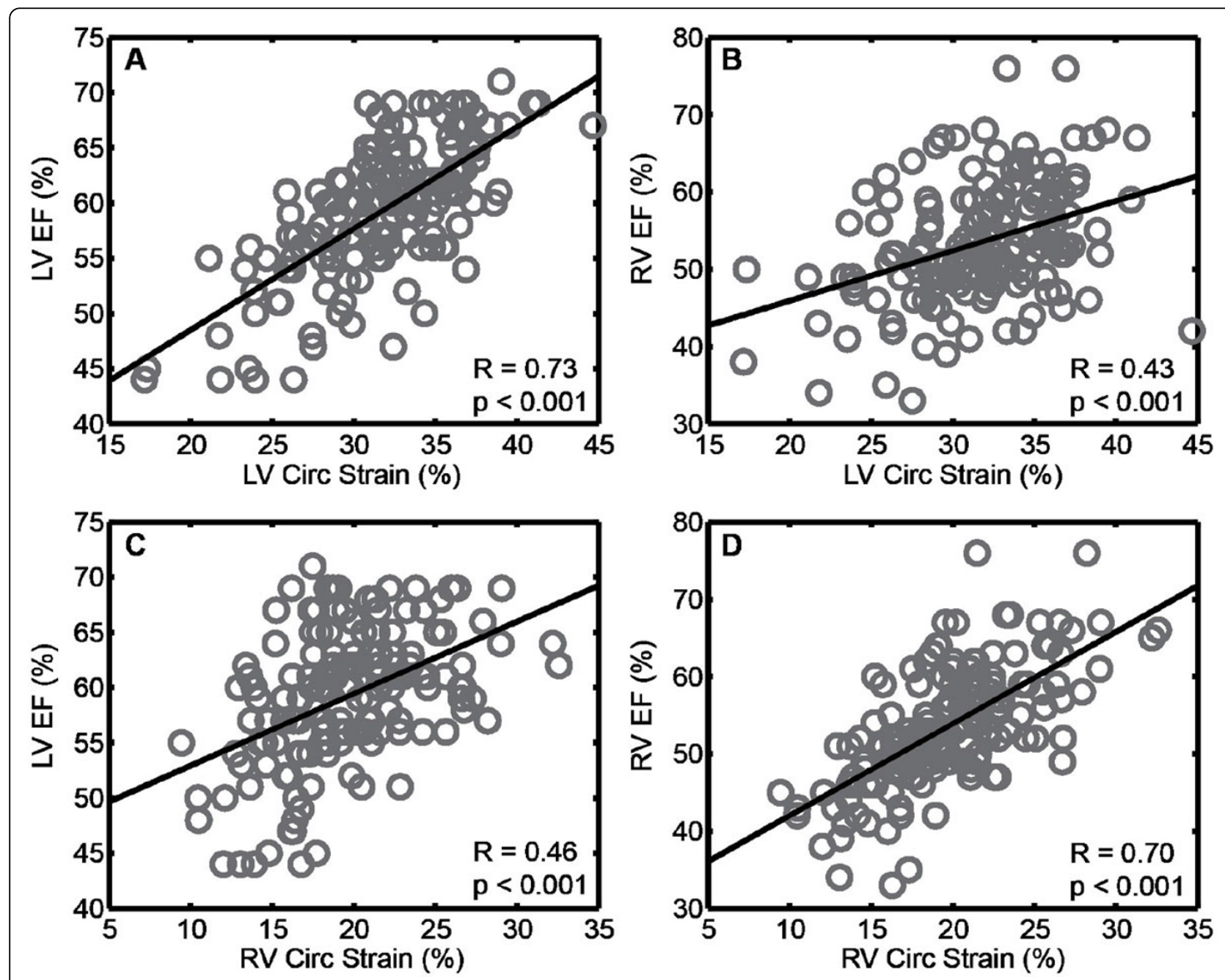

Figure 1 Correlation between peak circumferential strain and ejection fraction. A: LV circumferential strain vs LV EF; B: LV circumferential strain vs RV EF; C: RV circumferential strain vs LV EF; D: RV circumferential strain vs RV EF.

USA. ${ }^{4}$ Department of Cardiology, Boston Children's Hospital, Boston, MA, USA.

Published: 27 January 2016
doi:10.1186/1532-429X-18-S1-032

Cite this article as: Jing et al:: Association of ventricular dyssynchrony and strain with cardiac function in patients with repaired tetralogy of Fallot. Journal of Cardiovascular Magnetic Resonance 2016 18(Suppl 1):032. 